\title{
Thermal Desorption of Hydrogen at the Titanium Hydride-Oxide Interface
}

\author{
Bun Tsuchiya, Shinji Nagata, Naofumi Ohtsu, Kentaro Toh and Tatsuo Shikama \\ Institute for Materials Research, Tohoku University, Sendai 980-8577, Japan
}

\begin{abstract}
Changes in hydrogen distribution in $\delta$-phase titanium hydrides $\left(\mathrm{TiH}_{1.92 \sim 1.95}\right)$, covered with titanium oxide layers $(\mathrm{TiO}$ and $\mathrm{TiO} 2)$, were investigated by elastic recoil detection analysis (ERDA) after isochronal and isothermal annealing experiments. The hydrogen concentration near to the surface of the hydride exponentially decreased as a function of annealing time in the temperature range from 423 to $523 \mathrm{~K}$. The result shows that the thermal desorption of hydrogen from the hydride is governed by first-order reaction kinetics and greatly depends on the thermal detrapping process. The thermal detrapping rates of hydrogen at the hydride-oxide interface were estimated and their activation energy was determined to be $1.3 \pm 0.2 \mathrm{eV}$.
\end{abstract}

(Received October 13, 2004; Accepted January 11, 2005)

Keywords: titanium hydride, titanium oxide, elastic recoil detection, thermal desorption, isothermal annealing, thermal detrapping

\section{Introduction}

The development of a hydrogen-air fuel cell consisting of proton-conducting oxides that act as a solid electrolyte and of hydrogen storage metals as a hydrogen source has been proposed by many workers. ${ }^{1,2)}$ This closed-type fuel cell, which does not require hydrogen gas to be supplied from outside, is promising as a compact electric power-generating system that would help to satisfy the growing demand for zero $\mathrm{CO}_{2}$-emitting energy sources. The rate of hydrogen transport to the fuel cell is one of the most important ratedeterminant processes in the closed-type fuel cell. It is extremely important to understand how hydrogen is transported from the hydrogen storage metals to the protonconducting oxides. Several reports have recently been published on the hydrogen diffusion, hydride dissociation and hydrogen evolution characteristics of titanium hydrides. $^{3,4)}$ However, the surface of the hydride specimens is clearly oxidized, and the correlation between hydrogen migration and the oxide layers is as yet not fully understood.

In the present study, depth profiles of hydrogen atoms in the surface of titanium hydrides after annealing have been measured using an elastic recoil detection analysis (ERDA) which is a form of non-destructive ion beam analysis method with various energies that is used to investigate the thermal behavior of hydrogen atoms at the interface between the hydride and the oxide layers and the fundamental phenomenon leading to emission of atoms from the solid.

\section{Experiments}

Nonstoichiometric titanium hydrides were fabricated by maintaining the equivalent conditions for 4 days at a hydrogen pressure of $1.0 \times 10^{5} \mathrm{~Pa}$ and a temperature of $1073 \mathrm{~K}$ using a Sieverts apparatus. The $\mathrm{H} / \mathrm{Ti}$ ratios of the hydride specimens were determined to be $1.92 \sim 1.95$ by mass gain, hydrogen pressure changes as well as hydrogen analysis, was analyzed by means of high-energy ERDA using $2.8 \mathrm{MeV}$ $\mathrm{He}^{2+}$ ions. 5) The hydride specimens were irradiated with $\mathrm{He}^{2+}$ ions at an incident angle of $75^{\circ}$ to the surface normal of the specimen at room temperature. The hydrogen atoms forward-recoiled by elastic collisions were detected at a scattering angle of $30^{\circ}$ to the incident ion direction. An absorber comprising $12 \mu \mathrm{m}$-thick $\mathrm{Al}$ film was installed in front of the ERDA detector. In addition, a Rutherford backscattering spectrometry (RBS) measurement was simultaneously performed to act as an ion fluence monitor, in which the backscattered $\mathrm{He}^{+}$ions were detected at an angle of $170^{\circ}$ to the incident ion direction. It was confirmed by Xray diffraction (XRD) measurement that the hydride had a face-centered cubic structure ( $\delta$-phase) at room temperature. It was also observed by X-ray photoelectron spectroscopy (XPS) and co-axial impact collision ion scattering spectroscopy (CAICISS) measurements with $\mathrm{Ar}^{+}$ion sputtering at room temperature (Fig. 1(a)) and isochronal annealing for $10 \mathrm{~min}$ at several temperatures up to $551 \mathrm{~K}$ (Fig. 1(b)) that $\mathrm{TiO}$ and $\mathrm{TiO}_{2}$ layers of about $10 \mathrm{~nm}$ thick were formed as the topmost surface on the hydride and were stable in this temperature region. The presence of hydrogen in the hydride phase has a considerable influence on oxidation dynamics, and the oxidation process takes place only on the hydride surface. ${ }^{6)}$ Moreover, a small amount of water, absorbed on the topmost surface, was observed by direct collision with the impinging primary ions, based on topmost surface-sensitive techniques consisting of CAICISS and low-energy ERDA using $2.0 \mathrm{keV} \mathrm{He}^{+}$ions. ${ }^{7)}$ The hydride specimens were irradiated with the pulsed $\mathrm{He}^{+}$ions at an angle of $0^{\circ}$ to the surface normal of the specimens. The backscattered $\mathrm{He}^{+}$ions were detected at an angle of $180^{\circ}$ to the incident ion direction by time of flight (TOF) using a co-axial micro channel plate (MCP) detector. In the low-energy ERDA measurement case, the hydride specimens were irradiated with the pulsed $\mathrm{He}^{+}$ ions at an angle of $79^{\circ}$ to the surface normal of the specimens. The hydrogen atoms that forward-recoiled at a scattering angle of $34^{\circ}$ to the incident ion direction were detected by TOF with an MCP detector for ERDA. A small quantity of water remained on the topmost oxide layers after heating at $573 \mathrm{~K}$ for $10 \mathrm{~min}$.

To investigate the thermal behavior of hydrogen at the hydride-oxide interface and hydride bulk, the hydrogen concentration in the hydride was measured by high-energy ERDA after isochronal annealing for $10 \mathrm{~min}$ at several temperatures to $573 \mathrm{~K}$, and isothermal annealing in the temperature range from 423 to $523 \mathrm{~K}$ were carried out. The temperature of the specimens was measured with an alumelchromel thermocouple in contact with the surface. 

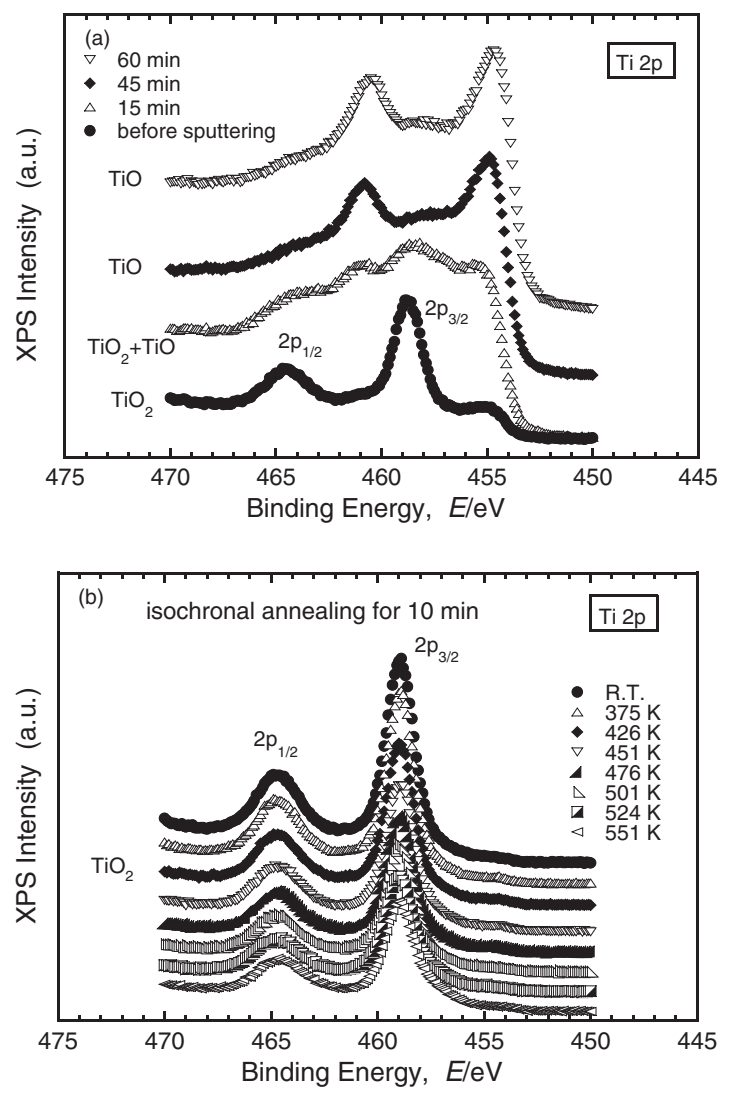

Fig. 1 XPS spectra of $\mathrm{Ti} 2 \mathrm{p}$ after (a) $\mathrm{Ar}^{+}$ion sputtering at room temperature and (b) isochronal annealing for $10 \mathrm{~min}$ at several temperatures up to $551 \mathrm{~K}$.

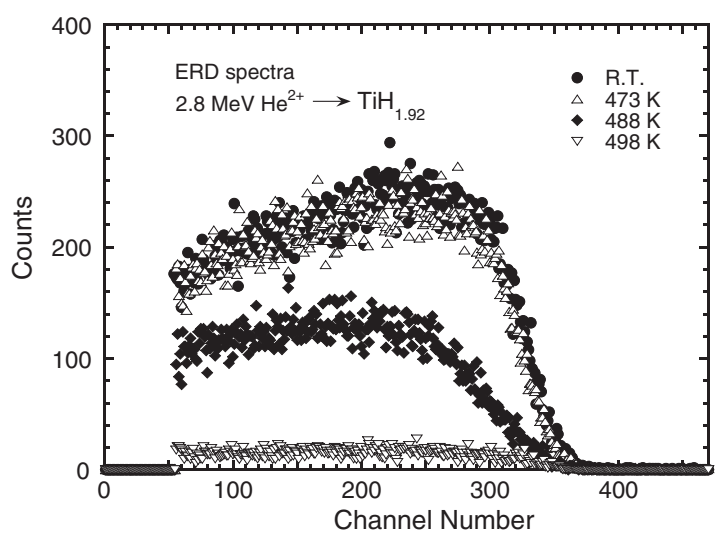

Fig. 2 High-energy ERDA spectra of $\mathrm{H}$ recoiled from $\mathrm{TiH}_{1.92}(\bullet)$ before and after isochronal annealing for $10 \mathrm{~min}$ at $(\triangle) 473 \mathrm{~K},(\diamond) 488 \mathrm{~K}$ and $(\nabla)$ $498 \mathrm{~K}$.

\section{Results and Discussion}

Figure 2 shows typical ERDA spectra of $\mathrm{H}$ recoiled from $\mathrm{TiH}_{1.92}$ after annealing at each temperature for $10 \mathrm{~min}$, measured using $2.8 \mathrm{MeV} \mathrm{He}^{2+}$ ion probe beams. The horizontal axis corresponds to several energies of the recoiled hydrogen atoms and represents the distance from the surface. The total integral counts of the ERDA spectra are almost the same until $398 \mathrm{~K}$, but rapidly fall from $473 \mathrm{~K}$. To obtain the depth profile, the averaged $\mathrm{H}$ counts to 10 channels in the ERDA spectra, the $\mathrm{He}^{+}$ion fluence, the elastic recoil cross-

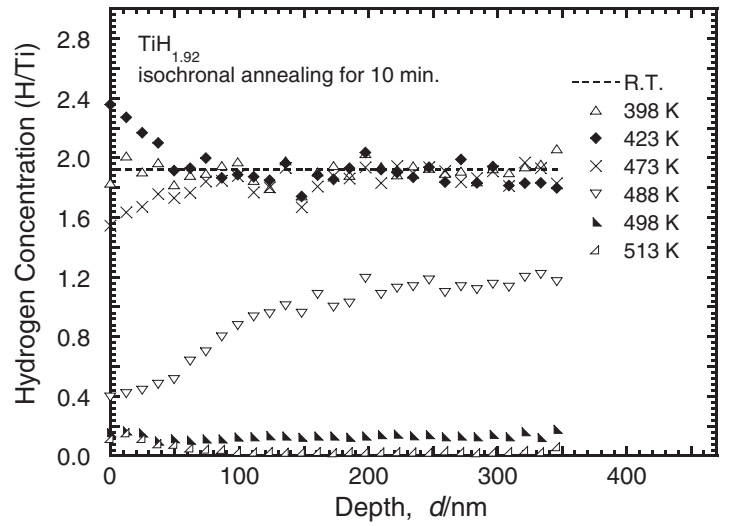

Fig. 3 Hydrogen distributions in the surface of $\mathrm{TiH}_{1.92}$ before and after isochronal annealing for $10 \mathrm{~min}$ at $398 \mathrm{~K}, 423 \mathrm{~K}, 473 \mathrm{~K}, 488 \mathrm{~K}, 498 \mathrm{~K}$ and $513 \mathrm{~K}$.

sections of $\mathrm{He}^{+}$ion for $\mathrm{H}$, the stopping cross-sections for $\mathrm{H}^{+}$ and $\mathrm{He}^{+}$ions in $\mathrm{TiH}_{\mathrm{x}}$ and the solid angle of the detection used were taken into account. The $\mathrm{He}^{+}$ion fluence, calculated from standard analysis of the RBS spectrum, was constant for all ERDA measurements. The stopping cross-sections change little, even if the hydrogen concentration decreases, because the stopping powers of $\mathrm{H}_{\text {for }} \mathrm{H}^{+}$ and $\mathrm{He}^{+}$ions are much smaller than those of $\mathrm{Ti}$ for $\mathrm{H}^{+}$and $\mathrm{He}^{+}$ions. Although the elastic recoil cross-section varies according to the change in incident $\mathrm{He}^{+}$ion energy against the depth, the values are almost the same for all ERDA spectra that have been obtained after heating at several temperatures. Therefore, in the present study, the distribution of $\mathrm{H}$, retained to around $300 \mathrm{~nm}$ deep in $\mathrm{TiH}_{1.92}$, was estimated by normalizing the ERDA spectra from several temperatures to that at room temperature and then plotted in Fig. 3. It is of particular interest that the hydrogen concentration close to the surface changed to a much greater extent at $423 \sim 513 \mathrm{~K}$ than in the bulk. The hydrogen concentration to about $50 \mathrm{~nm}$ deep increased at $423 \mathrm{~K}$ but decreased at $473 \mathrm{~K}$. The amount of $\mathrm{H}$ retained in the bulk at $488 \mathrm{~K}$ was higher than that in the surface. The depth profiles clearly show hydrogen migration. The hydrogen concentration gradient shows that hydrogen atoms, detrapped from the tetrahedral trap sites, are released with diffusion to the surface and hydrogen molecular recombination on the topmost surface. On the other hand, at $513 \mathrm{~K}$, the hydrogen concentration near to the surface less than $50 \mathrm{~nm}$ in depth exceeded that in the bulk. It was found by low-energy ERDA combined with CAICISS that the increased presence of hydrogen at the surface can be ascribed to absorption of water on the uppermost oxide layers.

To identify the mechanism of the thermal re-mission of hydrogen from $\delta$-phase $\mathrm{TiH}_{1.95}$, isothermal annealing experiments were performed at $423,473,488,493$ and $523 \mathrm{~K}$. The isothermal re-emission curves below $100 \mathrm{~nm}$ in depth at these temperatures are summarized as a function of annealing time in Fig. 4, where the vertical axis represents the retained number normalized by the number of $\mathrm{H}$ in $\mathrm{TiH}_{1.95}$ before each annealing. The hydrogen concentrations at several temperatures decrease with increasing annealing time, and their decay rates increase as the annealing temperature 


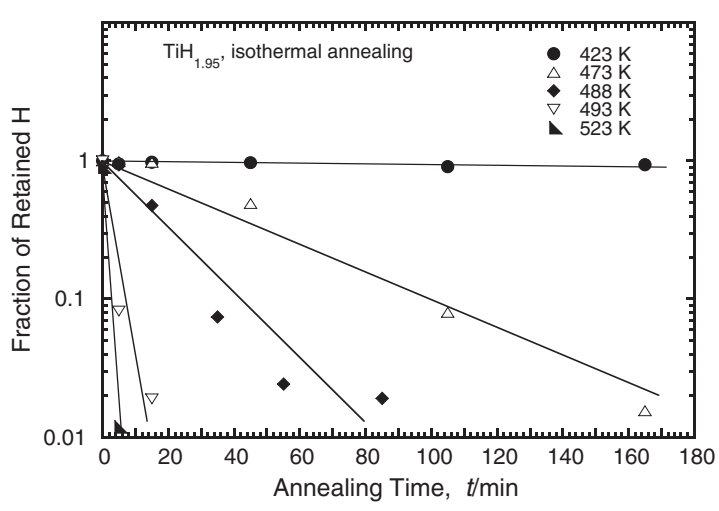

Fig. 4 Changes in hydrogen concentration near the surface below $100 \mathrm{~nm}$ in depth of $\mathrm{TiH}_{1.95}$ as a function of annealing time at 423 to $523 \mathrm{~K}$.

increases. The entire isothermal annealing curves can be approximated by one exponential function, as shown by the solid lines, since the vertical axis in Fig. 4 is scaled logarithmically. This result indicates that the thermal desorption of hydrogen from $\mathrm{TiH}_{1.95}$ can be described by first-order reaction kinetics and greatly depends on the thermal detrapping process, since hydrogen release takes place through molecular recombination on the topmost surface after thermal detrapping of hydrogen from the trap sites followed by diffusion to the surface. Thus, the decay rate of the hydrogen concentration corresponds to the thermal detrapping rate. The thermal detrapping rates at several temperatures were determined from the slope of the solid lines in Fig. 4 and are plotted in kelvin as a function of $1000 / T$ in Fig. 5. As a result, the activation energy of the thermal detrapping rate was determined to be $1.3 \pm 0.2 \mathrm{eV}$ from the slope of the solid line in Fig. 5. The activation energy is greater than that of $0.55 \mathrm{eV}$ for the previously reported diffusion rate. ${ }^{3)}$ Therefore, the activation energy may correspond to the potential that is associated with intense lattice distortion at the interface between the hydride and oxide layers.

The oxide layers play an important role as surface barriers for hydrogen retention in hydrogen storage metals, since they impede hydrogen transport. Since the hydrogen desorption from the hydrides is controlled by the oxide layers, it will be

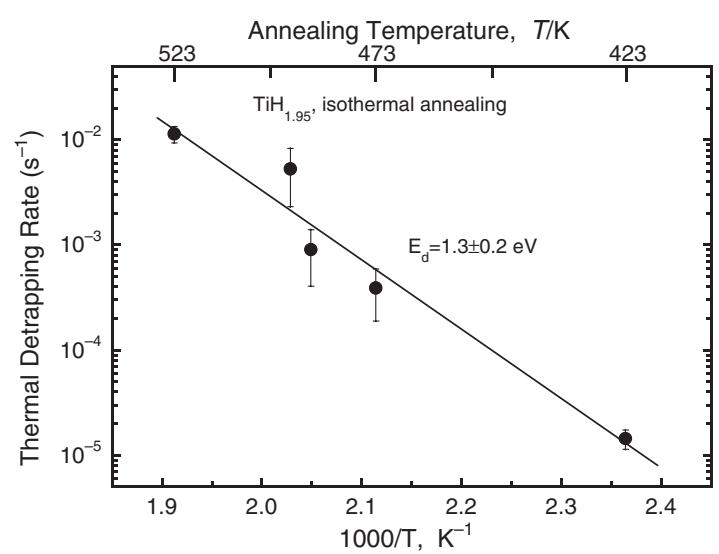

Fig. 5 Arrhenius plots of thermal detrapping rates of hydrogen in $\mathrm{TiH}_{1.95}$ at 423 to $523 \mathrm{~K}$ necessary to use various techniques to modify the hydrideoxide interface to further enhance hydrogen transport from the hydrogen storage metals to the proton-conducting oxides and enable the process to take place at a lower temperature.

\section{Summary}

The $\delta$-phase $\mathrm{TiH}_{1.92 \sim 1.95}$ specimens were prepared by adjusting the gas pressure and the temperature by means of a Sieverts apparatus. It was confirmed by XPS and CAICISS, combined with low-energy ERDA, that the hydrides were covered with titanium oxide layers about $10 \mathrm{~nm}$ thick, and, moreover, a small quantity of water was dissociated on the oxide topmost surface. After isochronal annealing at several temperatures to $573 \mathrm{~K}$ for $10 \mathrm{~min}$, the changes in the hydrogen distribution near to the surface (to about $300 \mathrm{~nm}$ deep) of the hydride were investigated using high-energy ERDA. The re-emission of hydrogen from the hydride started at $473 \mathrm{~K}$. Hydrogen migration and release were clearly shown in the depth profiles.

To clarify the thermal behavior of hydrogen from the hydride to the oxide layers, isothermal annealing experiments were carried out in the temperature range of 423 to $523 \mathrm{~K}$. The isothermal re-emission curves at each temperature were approximated by one exponential function. This fact suggests that the thermal re-emission of hydrogen from titanium hydride is governed by first-order reaction kinetics. Notably, the thermal detrapping of hydrogen from the trap sites plays the most important role in the thermal release. The thermal detrapping rates were estimated and their activation energy was determined to be $1.3 \pm 0.2 \mathrm{eV}$, which was greater than that of $0.55 \mathrm{eV}$ for the diffusion rate, from analysis of the reemission curves. Therefore, the activation energy may correspond to the potential energy at the surface barrier, and the hydrogen migration rate may depend primarily on the characteristics of the hydride-oxide interface.

\section{Acknowledgements}

The authors are indebted to Dr. M. Asari, Mr. D. Imai and Dr. H. Nakamura for the experimental results of the CAICISS and low-energy ERDA, which were supported by Shimadzu Corporation.

\section{REFERENCES}

1) C. Folonari, G. Lemmi, F. Manfredi and A. Rolle: J. Less-Common Met. 74 (1984) 371-378.

2) S. Yamaguchi, H. Yugami and S. Ikeda: J. Alloys Comp. 330-332 (2002) 911-915.

3) U. Kaess, G. Majer, M. Stoll, D. T. Peterson and R. G. Barnes: J. Alloys Comp. 259 (1997) 74-82.

4) A. Takasaki, Y. Furuya, K. Ojima and Y. Taneda: J. Alloys Comp. 224 (1995) 269-273.

5) B. Tsuchiya, M. Teshigawara, S. Nagata, K. Konashi, R. Yasuda, Y. Nishino, T. Nakagawa and M. Yamawaki: Nucl. Instr. and Meth. in Phys. Res. B 190 (2002) 699-703.

6) A. R. Gromov, N. N. Kouznetsova, S. L. Yudina and V. V. Lunin: J. Alloys Comp. 261 (1997) 269-272.

7) K. Konishi, S. Ogura, N. Ikuchi, D. Imai, T. Nishihara and M. Shinohara: Diamond and Related Materials 9 (2000) 746-751. 